Didáctica. Lengua y literatura

ISSN: $1130-0531$

http://dx.doi.org/10.5209/DIDA.61962

\title{
Evaluación del proceso de tutorización del TFM de la Facultad de Filología de la UNED con metodología de educación a distancia
}

\author{
Nuria Polo Cano ${ }^{1}$; Inmaculada Senra Silva²; Araceli Gómez Fernández ${ }^{3}$
}

Recibido: 6 de noviembre de 2017 / Aceptado: 15 de junio de 2018

Resumen. En el campo de las filologías se ha detectado una carencia significativa con respecto a la metodología de trabajo para la tutorización a distancia del Trabajo Final de Máster (TFM), que es uno de los requisitos para la obtención del título, según la normativa sobre el Espacio Europeo de Educación Superior. El objetivo de este proyecto es realizar un análisis exhaustivo y a gran escala de las necesidades y opiniones de los profesores que participan en once másteres de la Facultad de Filología de la UNED con respecto a la tutorización y evaluación de los trabajos finales. A través de un cuestionario se recaba información de los profesores sobre su experiencia en todo el proceso: la temática de los trabajos, la cantidad de tiempo invertido, las tareas realizadas durante la tutorización, la evaluación del TFM, la respuesta de los estudiantes, etc. Los datos indican la pertinencia de contar con directrices generales válidas para todos los másteres que agilicen el proceso de tutorización y faciliten la evaluación del TFM, de manera que el tutor vea rentabilizados el tiempo y esfuerzo invertidos. Además, los profesores reclaman mejoras institucionales y el aumento de la autonomía y el rigor académico a los estudiantes, quejas que no nos diferencian de otras universidades y otros contextos educativos.

Palabras clave: Trabajo fin de máster (TFM); Filología; tutorización; evaluación; enseñanza a distancia.

\section{[en] Evaluation of the Tutoring in a Distance-Learning Context of Masters Dissertations at the UNED Faculty of Philology}

\footnotetext{
Abstract. In the field of Modern Languages, a significant shortfall in methodological guidance for distance tutoring of students' Master's dissertations (TFM) - one of the requirements for obtaining the degree, in line with European Higher Education Area regulations - has been observed. The objective of this project is to carry out a large-scale analysis of the needs and opinions of the teachers participating in the eleven Master courses of the Faculty of Philology at the UNED in relation to the tutoring and assessment of the TFM. By means of an online questionnaire, information will be collected from the teachers about their experience throughout the process: the topics, the amount of time invested, the tasks performed during the tutoring process, assessment/evaluation of the TFM, students' responses etc. The data collected show the importance of having general guidelines which will be valid for all masters and which will speed up the process of tutoring and facilitate the evaluation of the TFM, so

1 Departamento de Lengua Española y Lingüística General. Universidad Nacional de Educación a Distancia (España)

E-mail: nuriapolo@flog.uned.es

2 Departamento de Filologías Extranjeras y sus Lingüísticas. Universidad Nacional de Educación a Distancia (España)

E-mail: isenra@flog.uned.es

3 Departamento de Filología Francesa. Universidad Nacional de Educación a Distancia (España)

E-mail: aragomez@flog.uned.es
} 
that the tutors' work becomes more effective. In addition, teachers demand institutional improvements and increased autonomy and academic rigor from students, complaints that do not differentiate us from other universities and other educational contexts.

Keywords: Master Thesis (TFM); Philology; tutoring; evaluation; distance learning.

\section{[fr] Évaluation du processus de direction du MFM de la Faculté de Philologie de l'UNED avec la méthodologie d'enseignement à distance}

Résumé. Dans le domaine de la philologie, un manque significatif dans la direction à distance du Mémoire Final du Master (MFM) a été détecté par rapport à la méthodologie de travail. Conformément aux dispositions de l'Espace Européen de l'Enseignement Supérieur, le MFM est indispensable pour l'obtention du diplôme de master. L'objectif de ce projet est de réaliser une analyse exhaustive et à grande échelle des besoins et des opinions des enseignants participant aux onze Master de la Faculté de Philologie de l'UNED vis-à-vis de la direction et de l'évaluation des mémoires de master. L'information sur l'expérience des enseignants est recueillie grâce à un questionnaire tout au long d'un processus: sujet du travail, le temps consacré, les tâches accomplies pendant la direction, l'évaluation du mémoire de master, la réponse des étudiants, etc. Les données indiquent la pertinence des lignes directrices générales valables pour tous les masters. Celles-ci accélèrent le processus de direction et facilitent l'évaluation du mémoire, de sorte que le directeur voit son temps et ses efforts investis rentabilisés. En outre, les enseignants exigent des améliorations institutionnelles et aux étudiants une plus grande autonomie et rigueur académique. Ce sont des besoins communs aux autres universités et aux autres contextes éducatifs.

Mots-clés: Mémoire fin de master (MFM); Philologie; direction; évaluation; enseignement à distance.

Sumario. 1. Introducción. 2. Objetivos. 3. Método. 4. Resultados. 4.1. La cantidad de trabajo y el tiempo empleado. 4.2. La temática. 4.3. Las tareas de tutorización. 4.4. La evaluación del trabajo. 4.5. La actuación de los profesores en los tribunales de defensa. 4.6. Los trámites administrativos. 4.7. La actuación del estudiante durante la elaboración y la defensa del TFM. 4.8. Comentarios finales. 5. Conclusiones. 6. Bibliografía.

Cómo citar: Polo Cano, N.; Senra Silva, I.; Gómez Fernández, A. (2018) Evaluación del proceso de tutorización del TFM de la Facultad de Filología de la UNED con metodología de educación a distancia, Didáctica. Lengua y Literatura, 30, 185-199.

\section{Introducción}

En la Facultad de Filología de la UNED, desde 2008, se imparte un conjunto de másteres oficiales, tanto de orientación lingüística como literaria, acogidos a la normativa vigente sobre el Espacio Europeo de Educación Superior (EEES). Uno de los requisitos para la obtención del título es la realización de un Trabajo Fin de Máster (TFM), tutelado y dirigido por un profesor con docencia en los mismos, con una carga docente de 10 créditos ECTS (European Credit Transfer System), esto es, unas 125 horas de dedicación. Se espera que, tras la corrección y maduración de varios borradores, el estudiante alcance las destrezas necesarias para desarrollar su primer trabajo de investigación que, además, supone un entrenamiento de lo que posteriormente podría ser una investigación de mayor calado como la tesis doctoral, en caso de que se decida a realizarla. 
En el campo de las filologías se ha detectado una carencia significativa con respecto a la metodología de trabajo para la tutorización de los TFM que llevan a cabo los docentes, especialmente importante en una universidad a distancia como la nuestra. La UNED se caracteriza por una enseñanza a distancia, por lo que en la mayoría de ocasiones el estudiante no acude, porque no vive en la misma ciudad, a reuniones presenciales con su tutor y toda la corrección del trabajo se realiza por teléfono (o Skype) y por correo electrónico. A esto hay que añadir que el profesor no conozca a ese estudiante, porque nunca le haya dado clase previamente. Por ello, tanto la distancia como el no conocimiento previo pueden dificultar la tarea de la elaboración de un TFM. Además, en el caso específico de la filología, especialmente en el caso del español, puede ocurrir que mucha bibliografía (por ejemplo, fondos antiguos) solo se encuentre en Madrid o en bibliotecas españolas y el estudiante no esté en disposición (física) de consultarlos.

Este trabajo se enmarca dentro de un proyecto mayor en el que se analiza la visión de los estudiantes respecto del TFM (San Mateo Valdehíta, Escobar Álvarez y Chacón Beltrán, en prensa), visión que es la que normalmente se analiza. Por el contrario, la perspectiva del profesor ha sido abordada anteriormente por otros equipos de trabajo en el ámbito nacional en estudios muy recientes, aunque en otras áreas de conocimiento o en otros contextos educativos que no implican la educación a distancia (Cabrera, Rodera y Sangrà, 2012; Da Cunha, 2016; Fernández Sánchez et al., 2015; Miraflores Gómez et al., 2016; Pérez Navarro et al., 2013; Rodríguez et al., 2013; González Calero Somoza y Turégano Moratalla, 2011; Velayos Martínez et al., 2016).

Por este motivo, se ha considerado la pertinencia de analizar las necesidades académicas atendiendo a nuestro contexto específico de enseñanza/aprendizaje a distancia, con el fin de poder evaluarlas mediante diferentes propuestas que faciliten el trabajo y rentabilicen los esfuerzos del personal docente.

\section{Objetivos}

El objetivo principal de este proyecto es realizar un análisis exhaustivo, de tipo cualitativo y cuantitativo, de las necesidades y opiniones de los profesores de los distintos másteres de la facultad de Filología con respecto a la tutorización y evaluación de los trabajos finales, en la modalidad de educación a distancia, para detectar sus puntos débiles. Como objetivo secundario, y una vez identificados, se proponen las medidas oportunas para reforzarlos o en cierta medida mejorar la práctica docente que redundará en mejores resultados en los estudiantes. En definitiva, cabe preguntarse si la calidad de los másteres impartidos en la Facultad mejoraría gracias a un aumento de la motivación del profesorado y a una mejora en su dedicación hacia los estudiantes. De ahí la importancia de llevar a cabo esta investigación.

\section{Método}

La actividad se ha desarrollado en los once másteres de la Facultad de Filología. Se ha contado con los profesores que imparten docencia en estos másteres (ciento uno). 
Entre ellos, se incluyen los que forman parte de la plantilla de la UNED y los que proceden de otras universidades, pero que colaboran en los másteres de la universidad. Para conseguir una participación representativa se les ha informado de que las respuestas se usarán para rentabilizar su labor y de que el fin de la investigación es mejorar la calidad del trabajo. Los profesores que así lo han deseado se han identificado, de forma que no ha sido una participación anónima. Pero, anónima o no, la participación en el proyecto ha sido voluntaria.

Para recabar la información, se ha elaborado un cuestionario en línea. Ha sido confeccionado con un formulario de Google, de fácil creación, manejo y distribución por Internet. Esta herramienta además permite organizar las respuestas en una hoja de cálculo, que sirve para integrar la interpretación de las mismas.

Así pues, tras consultar la bibliografía y a partir de nuestra propia experiencia, hemos elaborado las preguntas, que se han organizado en varias secciones sobre los aspectos más relevantes del proceso de tutorización y calificación de un TFM: (1) la cantidad de trabajos y el tiempo empleado, 10 ítems, (2) la temática, 6 ítems, (3) las tareas de tutorización, 4 ítems, (4) la evaluación del trabajo, 4 ítems, (5) la actuación de los profesores en los tribunales de defensa, 8 ítems, (6) los trámites administrativos, 4 ítems, (7) la actuación del estudiante durante la elaboración, 8 ítems, y (8) la defensa del TFM, 6 ítems. Además de un campo para comentarios finales. En total, se ha presentado una encuesta integrada por cincuenta y una preguntas, cuyas respuestas se podían completar fácilmente en aproximadamente diez minutos.

Con el fin de recopilar las respuestas del mayor número de profesores de todos los másteres se ha contado con la colaboración de una Tutora de Apoyo en Red (TAR), que dispone de acceso a los cursos virtuales y tiene contacto con todos los profesores y estudiantes. De esta forma, dicha tutora fue la encargada de realizar la distribución del cuestionario. El equipo de trabajo se complementó con la participación de una tutora del Centro Asociado de Valencia que colabora en la implementación del cuestionario en el formulario de Google y en el análisis de los datos obtenidos.

\section{Resultados}

El resultado obtenido en la primera fase del trabajo ha sido el cuestionario mismo, que sirve para recopilar los datos de los tutores de los TFM, ya que en el proceso de elaboración de la encuesta hemos reflexionado y detectado los puntos débiles de la tutorización de un TFM. Efectivamente, la opinión de los docentes ha servido para corroborar en parte esas intuiciones iniciales y para confirmar los resultados de otras experiencias similares a esta citadas en la introducción. En este artículo se presentan los resultados de dicho cuestionario con el objetivo de utilizar la información relevante para mejorar la práctica docente tanto en nuestra universidad como en otras con problemas similares.

De entre los 101 docentes que integran los 11 másteres de la facultad de Filología, excluido el Máster en Formación del Profesorado, han participado 48 profesores, lo que supone una participación del 47,5\% y evidencia una alta participación con una buena representatividad (la muestra se corresponde con la mitad de la población). De estos 48 profesores, $42(87,5 \%)$ son profesores de la UNED, mientras que 6 $(12,5 \%)$ son profesores externos. En lo referente a la categoría profesional de los 
participantes en la encuesta, 4 (8,3\%) son catedráticos, 23 (48\%) titulares, $13(27 \%)$ contratados, $5(10 \%)$ ayudantes.

Como la participación fue voluntaria, no se podían prever ni la pertenencia ni la categoría de los participantes, así que la primera consideración en el análisis fue separar los datos por categoría profesional o por pertenencia interna a la UNED o externa a otras universidades para asegurar que estas dos variables no interfirieran en los resultados. Dicha separación no ha aportado diferencias significativas $(\mathrm{p}<0,05)$ a los resultados, es decir, los resultados no varían si la respuesta es dada por un titular o por un contratado; ni si el profesor es externo, aunque al ser esta participación muy baja, esta variable carece de representatividad. Por ello, se ha optado por presentar los resultados en el conjunto total de profesores.

\subsection{La cantidad de trabajos y el tiempo empleado}

El número de TFM dirigidos durante el curso 2016-2017 varía enormemente dependiendo del profesor. Por ejemplo, 9 profesores no han dirigido ningún TFM, mientras que 16 han dirigido entre 1-3 TFM, 18 profesores han tutorizado entre 4-6 trabajos y 5 profesores han dirigido más de 7 .

En este sentido, el $52 \%$ ( 25 profesores) de los encuestados opina que dicha cantidad de TFM es demasiada, el 35\% (17 profesores) cree que dicha cantidad es adecuada y el $12.5 \%$ ( 6 profesores) encuentra que es suficiente. Por lo tanto, si relacionamos la opinión sobre el número de trabajos dirigidos y la cantidad de trabajos realizados, obtenemos los siguientes datos: 17 profesores $(35,4 \%)$ piensan que dirigir entre 1 y 4 es adecuado, 2 profesores (4\%) consideran que más de 5 también es adecuado, pero 15 profesores $(31,5 \%)$ se ven sobrepasados con más de 5 , aunque $5(10 \%)$ lo están con 3. Por tanto, a partir de los datos descubrimos que el $30 \%$ de los profesores opina que su actual carga es injusta y que una cantidad más o menos aceptable de tutorizaciones de TFM por curso serían 4 trabajos como máximo por profesor.

En lo que respecta a la pregunta de si han notado que cada año dirigen un número mayor de TFM, el 48,9\% (23 profesores) ha respondido que no, mientras que el 51,1\% (24 profesores) ha respondido que sí. A raíz de estas respuestas se puede apreciar que estos porcentajes no parecen muy significativos.

En relación con el número de horas empleadas corrigiendo, leyendo o revisando el trabajo del estudiante, 2 profesores $(4,2 \%)$ han respondido que han necesitado menos de 10 horas, $16(33,3 \%)$ entre 10 y 20 horas y $30(62,5 \%)$ han indicado que más de 20 horas. De los 47 profesores que han respondido a esta pregunta, $20(42,6 \%)$ consideran que son demasiadas horas, $22(46,6 \%)$ estiman que son adecuadas, 3 $(6,4 \%)$ suficientes y $2(4,3 \%)$ creen que son insuficientes. Es decir, un $42,6 \%$ del profesorado siente que dedica más de 20 horas por TFM y considera que son demasiadas horas teniendo en cuenta el número de tutorandos por profesor.

Con respecto al número de horas empleadas atendiendo llamadas o respondiendo correos electrónicos, 21 profesores $(43,8 \%)$ han respondido que utilizan menos de 10 horas, $17(35,4 \%)$ han reconocido que entre 10 y 20 y 10 profesores $(20,8 \%)$ más de 20 horas. La valoración de esta cantidad ha dado lugar a $14(29,2 \%)$ profesores creen que ese número de horas es demasiado tiempo, 28 (58,\%) piensan que es adecuado, y $6(12,5 \%)$ suficiente. 
Por otro lado, el 70,8\% (34) de los encuestados considera que la universidad debería ofrecer un parámetro general del número de horas asignado a cada estudiante, mientras que el $29,2 \%$ (14) opina que no. Por último, casi el $70 \%$ cree que es necesario que el reparto del número de trabajos sea equitativo entre todos los profesores.

En lo que se refiere a la rentabilidad del tiempo invertido por estudiante, el 22,9\% (11) de los encuestados opina que esta ha sido alta, el 58,3\% (28) mantiene que ha sido media y el $16,7 \%$ (8) cree que ha sido baja. Solo un profesor considera que la rentabilidad ha sido insuficiente.

Con las respuestas obtenidas en estos apartados, podemos concluir que el tiempo estimado que cada tutor debe emplear por tutorando para responder llamadas y correos electrónicos debería estipularse en unas 10 horas, mientras que para leer y corregir el trabajo entre 10 y 20 horas. En general, el profesorado encuestado cree que se debería emplear alrededor de 30 horas totales por estudiante y TFM, nunca más de 40 horas. No obstante, si contamos el número máximo de trabajos recomendado por profesor, 4, esto supondría 160 horas de trabajo anual de un profesor dedicado únicamente a la dirección de TFM, es decir, algo como un mes de trabajo.

En definitiva, se ha constatado cuantitativamente un alto grado de saturación del profesorado. Por ello, entendemos que esta recomendación sobre el número máximo de trabajos por curso y el tiempo de horas invertidas debería aparecer en la Guía del Tutor que se elaborará como resultado de este estudio para poder rentabilizar el trabajo y evitar la saturación del profesorado de los distintos másteres. Asimismo, la universidad debería reflexionar sobre esta carga de trabajo entre su profesorado.

\subsection{La temática}

Sobre si la temática del trabajo se adecua a la línea de investigación del profesor, el $54,2 \%$ ( 25 profesores) ha respondido que siempre se adecua, el 41,7\% (20) ha contestado que a veces, y un 4,2\% (2) ha afirmado que casi nunca. La mitad del profesorado opina que estaría dispuesto a tutorizar un TFM que no correspondiera con su línea de investigación, mientras que la otra mitad opina que lo contrario.

Por otro lado, se vio necesario preguntar al profesorado si en algún momento había dudado de la autoría de los TFM tutorizados. En este sentido, el 60,4\% (29) respondió que no, mientras que el 39,6\% (19) respondió afirmativamente. Además, se preguntó si conocían o usaban alguna herramienta antiplagio. E1 37,5\% del profesorado indicó que contaba con herramientas para detectarlo, mientras que el 62,5\% no conoce o utiliza ningún tipo de ayuda para detectar estos casos. La mayoría utiliza el buscador de Google por no tener otras herramientas gratuitas a su alcance. Otras herramientas mencionadas son Turnitin, Grammarly, https://www.paperrater.com/ plagiarism_checker, http://www.plagium.com/, http://plagiarisma.net/es/ y http:// www.plagtracker.com/es/. El 97,9\% del profesorado encuestado considera que sería deseable que la universidad proporcionara herramientas antiplagio. A partir de estos resultados, se hace necesario, por tanto, solicitar a nuestra universidad que adquiera herramientas institucionales antiplagio y ponerlas de forma gratuita al servicio de los docentes 


\subsection{Las tareas de tutorización}

Acerca del papel que desempeña el tutor en la tutorización y evaluación de los TFM, cabe destacar que la mayoría de profesores $(70,8 \%)$ opina que no cuentan con información detallada. El 65,2\% de los encuestados no dispone de un documento de orientaciones para el tutor en su máster, mientras que el 34,8\% sí cuenta con algún documento de este tipo. Es esencial, por tanto, elaborar una Guía del Tutor con directrices concretas para poder homogeneizar esta labor entre todos los másteres de la Facultad.

A la pregunta abierta sobre los apartados o información que debería contener dicha guía, el 72,7\% ha contestado que deberían incluirse indicaciones para la gestión del tiempo, un 63,6\% opina que son necesarias plantillas de evaluación (rúbricas), un $75,8 \%$ propone la inclusión de unas preguntas frecuentes y un $75,8 \%$ cree conveniente contar con pautas sobre el seguimiento (por ejemplo, número de entregas) de la elaboración del trabajo. Entre otros posibles apartados, algunos encuestados (3\%) piensan que es necesario incluir la información que se considere necesaria para igualar la exigencia de los TFM entre los profesores, pues existe un sentimiento de que unos profesores son muy exigentes y otros no. También en este mismo apartado solicitan, en menor medida, directrices sobre la estructura y formato del TFM (3\%), que se incluyan indicaciones sobre la gestión administrativa del TFM (3\%), así como información detallada sobre las obligaciones del tutor y del tutorando $(3 \%)$ y un cronograma de trabajo (3\%).

Tras el análisis de las respuestas en este apartado, podemos concluir que la mayoría de profesores encuestados carece de directrices o documentos guía sobre cómo tutorizar un TFM y acomete la tarea confiando en su savoir faire. Precisamente por ello, consideran que necesitan información para una correcta u objetiva tutorización de los TFM. Se hace necesario, por tanto, elaborar una Guía del Tutor del TFM en nuestra facultad que homogeneice el proceso.

\subsection{La evaluación del trabajo}

En relación con la evaluación de los TFM, se preguntó a los profesores si contaban con plantillas de evaluación (rúbricas) tanto para el trabajo escrito como para la defensa oral. El 74,5\% respondió que no utilizaba plantillas de evaluación, mientras que el $25,5 \%$ contestó que sí contaba con rúbricas. En cuanto a la defensa oral, el $74,5 \%$ respondió que no disponían de plantillas para evaluar la defensa mientras que el $25,5 \%$ afirmó tenerlas. Ante esta falta de directrices, un $69,2 \%$ cree necesario contar con plantillas de evaluación de competencias del trabajo escrito, mientras que para la defensa oral, un $78 \%$ piensa que las rúbricas son necesarias. Como conclusión al análisis sobre la evaluación, un número elevado de profesores (entre el 70\% y $80 \%$ ) piensa que las rúbricas son aconsejables tanto para evaluar el trabajo escrito como para la defensa oral, aunque especialmente lo consideran así para la defensa oral. Por tanto, es recomendable diseñar unas rúbricas de evaluación, especialmente en el caso de las defensas orales, que puedan ser incluidas en la guía de orientaciones para el tutor. 


\subsection{La actuación de los profesores en los tribunales de defensa}

En el apartado de tribunales de defensa de los TFM, se preguntó a los encuestados si como miembros de dichos tribunales habían recibido los trabajos con el tiempo necesario para evaluarlos antes de la defensa oral. El 68,8\% respondió que siempre los había recibido con tiempo suficiente, aunque un $29,2 \%$ indicó que casi siempre y solo un $2,1 \%$ respondió que casi nunca.

Asimismo, se les preguntó si como miembros de un tribunal siempre habían juzgado trabajos cuya temática estaba relacionada con su área de investigación. El $37.5 \%$ respondió que siempre, el $60,4 \%$ que a veces y un $2,1 \%$ que casi nunca. Llama la atención que un $60,4 \%$ de los encuestados a veces juzgue trabajos que no tienen relación con su área de investigación.

En otro orden de cosas, un $41,7 \%$ de los profesores considera necesario que un docente de otro departamento forme parte del tribunal evaluador, mientras que un $58,3 \%$ piensa lo contrario. Aquellos encuestados que han respondido afirmativamente apuntan a que contar con un profesor de otro departamento aporta un punto de vista diferente, más objetivo, y puede ofrecer más garantías de imparcialidad. También opinan que es importante contar con un profesor externo especialmente cuando el trabajo es interdisciplinar. En este sentido, otros encuestados responden que en su departamento no hay profesores suficientes para formar parte de los tribunales y por ello necesitan profesores de otros departamentos y, en definitiva, disponer de profesores externos al departamento no aporta nada sustancial. Aunque una de las razones que más peso tiene en sus respuestas es que es muy difícil contar con compañeros de otros másteres que quieran o puedan formar parte del tribunal, por la carga de trabajo que ello conlleva o porque se aleja de sus áreas de investigación.

Por el contrario, los encuestados que opinan que no deberían formar parte de los tribunales profesores de otros departamentos lo justifican de distintas maneras: unos piensan que cada departamento establece unos baremos diferentes para juzgar los trabajos; otros que la exigencia de un profesor de otro departamento suele ser menor y que en ocasiones solo pueden opinar de la cuestión formal del TFM. Entre estos que están en contra, especialmente en el caso concreto del Máster en Lingüística Inglesa Aplicada, contar con profesores de otros departamentos que no hablan inglés supone un problema añadido, ya que los estudiantes no pueden redactar sus trabajos en dicha lengua.

Asimismo, se les preguntó si se debería establecer criterios para que todos los profesores de máster participen con el mismo número de TFM en los tribunales de defensa. El 66,7\% respondió positivamente, mientras que un 33,3\% respondió que no. Entre las razones de los primeros está la necesidad de que haya equidad en la carga docente de todos los profesores; la distribución equitativa del trabajo es una repuesta recurrente en varios apartados. Entre estos argumentos también se menciona la necesidad de que todo el profesorado esté involucrado y que se entienda que es una tarea y responsabilidad de todos.

Por otro lado, se les preguntó si alguna vez habían asistido a una defensa por videoconferencia y, en ese caso, si los medios habían sido los adecuados. De los 43 profesores que respondieron a esta pregunta, el 74,4\% respondió afirmativamente, mientras que el $25,6 \%$ dijo que no. Los primeros explicaron que las videoconferencias no funcionaron bien por problemas con la conexión en un $23 \%$ de los casos o por la mala calidad del sonido y la imagen tanto en Skype como en la plataforma 
AVIP. Además, los encuestados se quejan de que en general no hay técnicos de apoyo que ayuden y solucionen los problemas técnicos del momento, ni en la Sede Central ni en los Centros Asociados de la UNED. E incluso ha habido problemas con la conexión con centros en el extranjero.

Como conclusión a este apartado se puede afirmar que hay partes dentro del proceso de tutorización y defensa que escapan al propio control de los profesores y que se podrían mejorar desde la universidad o desde la facultad, como es el caso del mal funcionamiento de las videoconferencias o de la tutorización de trabajos cuya temática se aleja del área de investigación del tutor. Por ello, son necesarias propuestas de mejora para poder pedírselas a la universidad. Asimismo, es necesario un compromiso de entrega del TFM al profesor para poder leerlo con tiempo antes de la defensa oral y mejorar el reparto equitativo de los TFM.

\subsection{Los trámites administrativos}

En este apartado se preguntó a los profesores si consideraban adecuados los plazos de entrega y defensa del TFM (junio, septiembre y febrero). Las respuestas indican que el $77,1 \%$ está satisfecho con los plazos, mientras que un $22,9 \%$ no lo está. En general, consideran correctos los plazos actuales, aunque hacen algunas matizaciones. Por ejemplo, un profesor explica que los plazos son los mismos que para cualquier examen. Sin embargo, se debería regular el tipo de tutorización cuando el estudiante se presente en convocatoria extraordinaria, porque un profesor no debería estar tutorizando a una persona a lo largo de tres convocatorias desde que se matricula. Otro profesor opina que con las convocatorias de junio y septiembre debería ser suficiente; otro que los plazos de entrega deberían ser siempre durante el periodo lectivo, mientras que otro apunta que sería más operativa la entrega en junio y diciembre. Alguien explica que desde que se les asigna los TFM, los estudiantes cuentan en ocasiones con poco tiempo para realizarlos, menos de 4 meses si los quieren presentar en junio. Además, el plazo de septiembre es muy ajustado teniendo en cuenta que agosto y la primera semana de septiembre son periodos no lectivos. En este sentido, otro profesor considera que el plazo de septiembre tendría ser posterior (octubre) porque muchos estudiantes dedican el verano a acabar el TFM. Como no es lectivo, los tutores no pueden hacer el seguimiento, por lo que estaría bien aplazar la fecha de entrega. Todo esto conlleva que el TFM sea de facto una asignatura anual, cuando no es así, ya que actualmente solo se contempla lectivamente en el segundo cuatrimestre. No obstante, el número de convocatorias y los plazos vienen determinados por la universidad y por el calendario académico, por lo que hay poco que los profesores podamos decir.

En lo que respecta a la pregunta sobre si se debería simplificar y flexibilizar la gestión administrativa de los TFM, el 53,5\% de los 43 profesores que respondieron a esta pregunta dijo que sí, mientras que el resto lo hizo negativamente. En lo relativo a las propuestas de mejora para simplificar la gestión administrativa, algunas fueron:

- Defensa de los TFM en cualquier momento del curso cuando los tuvieran terminados, aunque la nota se pusiera en la convocatoria respectiva.

- Adelanto de los plazos de matrícula para que así se puedan nombrar los tutores antes de las vacaciones de Navidad y para que los estudiantes puedan fijar el 
tema con su tutor cuanto antes, ya que, si la matrícula del TFM se cierra en febrero, a menudo no hay tiempo para que el estudiante acabe el trabajo para la convocatoria de junio.

- Incorporación de la firma digital en la gestión administrativa del TFM para simplificar el proceso.

- Elaboración de guías que aclaren al estudiante los pasos a seguir.

- Gestión totalmente electrónica. Entrega a través de tareas en la plataforma virtual y que se elimine la entrega del trabajo en papel del trabajo.

- Disminución de la carga administrativa para el profesor.

En general, se puede observar que los profesores demandan una gestión íntegramente en formato digital, una reducción de la carga administrativa y el consiguiente aumento del tiempo para trabajar en el TFM. Como conclusión, para mejorar la labor de tutorización de TFM se hace necesario que desde la universidad se modifiquen algunos de los planteamientos actuales referidos a la gestión digital y a la carga administrativa, que escapan a comportamientos individuales de los docentes.

\subsection{La actuación del estudiante durante la elaboración y la defensa del TFM}

En lo que concierne a las obligaciones del estudiante que realiza un TFM, la mayoría de profesores responde que ellos, los docentes, sí las conocen, pero son los estudiantes los que no tienen claro en qué consiste la elaboración de un trabajo de estas características.

Los profesores señalaron que entre las tareas en las que suelen ayudar más a los estudiantes están, por orden de importancia, la explicación de la metodología (43 respuestas), la corrección de la redacción y del formato (42 respuestas), la orientación bibliográfica (41 respuestas), la selección del tema (36 respuestas), la estructuración del marco teórico ( 29 respuestas), la elaboración de las conclusiones (28 respuestas), el análisis metodológico/estadístico ( 25 respuestas), la presentación del material (1 respuesta) y el diseño de la experimentación (1 respuesta).

En general, según los encuestados, el nivel de conocimiento mostrado por los estudiantes durante la elaboración del TFM es medio (72,9\%), un 14,6\% indica que es bajo y un $4,2 \%$ que es muy bajo. Solo un $8,3 \%$ cree que el nivel de los estudiantes es alto.

En lo que se refiere al grado de madurez demostrado por los estudiantes, el 14,9\% piensa que es alto, el $63,8 \%$ considera que es medio, otro $14,9 \%$ que es bajo, y un $6,4 \%$ piensa que es insuficiente.

La mayoría del profesorado opina que el nivel de autonomía manifestado por los estudiantes para realizar el trabajo es medio $(56,5 \%)$, un $21,7 \%$ piensa que es bajo, un $8,7 \%$ insuficiente, y solo un $13 \%$ cree que es alto.

Según los encuestados, al inicio del TFM el estudiante debería mostrar, por orden de importancia, búsqueda de recursos bibliográficos (47 respuestas), dominio de una redacción académica (46 respuestas), capacidad de síntesis (45 respuestas), conocimientos metodológicos (programas informáticos, estadística, etc.) (37 respuestas), conocimiento de la materia (1 respuesta), qué es un TFM y qué se espera del estudiante (1 respuesta), gestores bibliográficos (1 respuesta) y técnicas de análisis de datos (1 respuestas). 
Además, los profesores demandan de los estudiantes que sean más autónomos y maduros, que gestionen bien su tiempo y cumplan los plazos; que sean más rigurosos en su trabajo intelectual y comprendan la labor investigadora que conlleva un TFM y que, por supuesto, el trabajo sea individual y original. Si los profesores están pidiendo esto es porque no reconocen estas características en los tutorizados, lo cual es muy significativo, ya que el estudiante de máster de la UNED es un adulto, generalmente en activo.

El hecho de que la mayoría de estudiantes no viva en Madrid no parece ser un problema a la hora de acceder a la bibliografía. Así opina el 35,4\% de los encuestados, mientras que el $18,7 \%$ cree que ello casi nunca supone un problema y solo un $10,4 \%$ lo considera un inconveniente.

En el apartado sobre la actuación de los estudiantes durante la defensa oral del TFM, el $17,8 \%$ de los profesores opina que siempre realizan una buena defensa, mientras que el $82,2 \%$ opina que solo a veces. En cuanto a los errores identificados en las exposiciones orales, los profesores que han participado en tribunales mencionan la falta de competencia para hablar en público, la falta de capacidad de síntesis, la falta de desarrollo del discurso oral y lenguaje académico y registro formal, la mala gestión del tiempo asignado, la excesiva confianza en la calidad de su trabajo y la escasez de humildad. Todas ellas son cualidades requeridas que no se enseñan o practican durante la propia elaboración del TFM.

En general los profesores opinan que la defensa oral sí influye en la calificación final del TFM, aunque solo un 14,6\% mantiene lo contrario, porque creen que lo importante es el contenido del trabajo y solo un $2 \%$ cree que la defensa debería influir más. Los comentarios sobre la importancia de las defensas orales se resumen en que algunas deficiencias del trabajo se resuelven en la exposición oral o en que la claridad expositiva garantiza el dominio, la madurez académica y la certeza sobre la autoría del trabajo. Por ello, el 93,5\% de los encuestados opina que las defensas orales presenciales no deberían eliminarse, mientras que un $6,5 \%$ cree que sí y que sería suficiente con una defensa por videoconferencia. Entre las razones para no suprimir la defensa presencial están las siguientes:

- La exposición presencial es más ágil y fluida y favorece la inmediatez del intercambio de opiniones entre el estudiante y el tribunal. (10 respuestas).

- Los medios técnicos de los que dispone la UNED y los Centros Asociados son en algunas ocasiones deficitarios como para suprimir las defensas presenciales. (5 respuestas).

- El estudiante debe tener un sentido del acto y de la institución. (2 respuestas).

Tras el análisis de estos datos, podemos concluir que los profesores están en general satisfechos con los conocimientos y con la madurez de los estudiantes, pero no con su grado de autonomía. Por otro lado, en no excesivas ocasiones existen problemas a la hora de acceder a la bibliografía cuando los estudiantes no viven en Madrid. En cuanto a la defensa oral, la mayoría del profesorado está satisfecho con su realización y opina que influye poco en la nota final del trabajo. Por ello, más del $90 \%$ de los encuestados no quiere suprimirla. En general, los profesores han descubierto carencias en los estudiantes sobre la falta de conocimientos de lo que debería ser una investigación y piensan que cuando los estudiantes llegan a la elaboración de un TFM deberían tener conocimientos previos sobre la búsqueda de recursos biblio- 
gráficos, la redacción y exposición académicas y la capacidad de síntesis. Estas tres capacidades, búsqueda bibliográfica, redacción-exposición y síntesis, deberían ser prerrequisitos para poder comenzar la realización del trabajo de estas características. Por ello, a la hora de elaborar una guía para el estudiante habrá que proporcionar pautas para mejorar su autonomía y el acceso a la bibliografía.

\subsection{Comentarios finales}

En la realización del cuestionario hemos considerado necesario incluir un apartado final abierto para que puedan añadir observaciones generales que los profesores consideren oportunas. Según algunos profesores, en general, hay estudiantes altamente preparados, a diferencia de quienes sienten que los estudiantes tienen grandes dificultades a la hora de sintetizar y redactar correctamente el TFM en términos académicos. Asimismo, algunos profesores han indicado que los estudiantes presentan carencias relacionadas con los conocimientos básicos teóricos del área en la que desean trabajar. Y especialmente en lo referido al nivel metodológico experimental y estadístico se observa una falta significativa de conocimientos común a todos los estudiantes.

La finalidad del TFM es la de formar al estudiante en habilidades de búsqueda de información, de capacidad de estructuración de las ideas que pretende desarrollar a nivel escrito y oral. Esta formación reforzará la autonomía del estudiante, ya que previamente ha realizado un TFG y conoce las bases de cómo realizar un trabajo. En el seguimiento del TFM se detectan errores, desvíos innecesarios o mal planteamiento del tema, pero hay que dar prioridad a una autonomía supervisada.

En definitiva, las labores de mejora o las propuestas de cambio se pueden resumir en los siguientes apartados:

- La unificación del trabajo: el profesorado considera que no existe homogeneidad a la hora de entender qué se debe exigir en un TFM.

- El seguimiento en las directrices del tutor: el estudiante ha de seguir su trabajo bajo los consejos del tutor, de tal forma que el visto bueno proporcionado por el tutor tenga un valor real.

- La formación del estudiante: este ha de tener claro qué es un TFM y cuáles son las normas de redacción del mismo. De esta forma se simplificaría el número de correcciones llevadas a cabo por el tutor referidas exclusivamente a qué es un TFM y no en el contenido del mismo. Esto podría mejorarse insistiendo más en las demás asignaturas del Máster, en la presentación de trabajos en estas asignaturas a nivel formal, estilístico y ortográfico.

- La formación estadística: no disponen de conocimientos de este tipo.

- El nivel dispar de los estudiantes: dirigir diferentes TFM permite comparar el grado de nivel de los estudiantes. Para evitar esta disparidad de nivel es necesario una mejora en la fase inicial del trabajo (cómo va a llevarse a cabo, qué se espera del tutor, número de entregas que ha de hacer el estudiante, etc.).

- Además, se ha constatado una bajada de nivel a lo largo de los últimos años, frente a los tradicionales DEA. Quizás pueda verse como un factor aleatorio, pero lo que sí es cierto es que el estudiante que realizaba un DEA antes estaba más encaminado hacia la investigación y mostraba un interés mayor. Se trata- 
ba de un estudiante con mayor autonomía y con un interés científico, a diferencia de hoy donde el TFM suele verse como un trámite y no como un inicio a su trayectoria investigadora. Así que parece que el desinterés mostrado por la investigación es proporcional al desconocimiento de las herramientas de trabajo para su realización. En definitiva, la evolución del concepto del TFM ha sufrido un cambio sustancial.

- La disparidad de intereses de los estudiantes conlleva una dispersión que obliga a ir de un tema a otro en estudiantes desconocidos por el equipo docente y a lo que hay que añadir la casuística personal de cada uno de ellos.

- Un consentimiento informado: los estudiantes deben conocer las tareas que deberán realizar desde el inicio. Han de dar su conformidad en el proceso de dirección y en la elección de su tutor, con conocimiento del bagaje académico de este y asumiendo las recomendaciones recibidas por él mismo. No se puede ni se debe hacer una tutorización defensiva en la que el estudiante imponga su criterio a pesar de los consejos del tutor.

- El tiempo invertido por el tutorando: el TFM es un trabajo realizado por el estudiante como una asignatura más y por ello ha de tener constancia del tiempo que necesitará para su realización y un buen resultado.

- El tiempo invertido por el profesor: el tutor es consciente del tiempo en la tutorización de un TFM teniendo en cuenta estas consideraciones. Se plantea el número de TFM que ha de asumir de manera que la dedicación con el estudiante y la calidad del trabajo sean lo más altas posibles. Deberían por lo tanto existir medios equitativos de reparto de estudiantes entre el profesorado.

- El tiempo de impartición del TFM: se trata de una asignatura cuatrimestral para que pueda ser cursada en un año, a sabiendas de que en la práctica pocos estudiantes, por no decir ninguno, superan esta asignatura en un curso académico, por lo que deberían plantearse titulaciones de dos años (la carga de trabajo de muchas asignaturas es claramente superior a la carga teórica de ECTS asignada) en las que el TFM sea una asignatura anual de entre 10 y 12 ECTS del segundo curso.

- La cronología de realización del TFM: al igual que se viene haciendo con el Trabajo de Fin de Grado, el estudiante debería contar con un cronograma que le permita realizar su TFM en fases que le llevaría a una mayor organización del trabajo. Esto permitiría al profesor un control de las horas que dedica a la tutorización del TFM.

- La guía de TFM es necesaria tanto para estudiantes como para profesores y ha de conllevar una mejora. Junto a ella, es necesaria también la elaboración de recursos documentales y audiovisuales.

A partir de estas consideraciones cabe destacar que algunos de los problemas que hemos detectado no son fáciles de subsanar, como es el caso del número asignado de TFM para dirigir por profesor, y que en ocasiones las soluciones se encuentran en el nivel institucional y no dependen meramente de la buena voluntad del profesor. El tema de la distancia es, sin embargo, consustancial a nuestra universidad, asumible por ambas partes y parece que no supone un problema en la elaboración de los trabajos 


\section{Conclusiones}

A partir de estos resultados se ha visto la necesidad de contar con unas directrices generales válidas para todos los másteres de la Facultad que agilicen el proceso de tutorización del TFM y faciliten su evaluación y, en definitiva, proporcionen cierta sistematización y homogeneización al proceso. Dichas indicaciones, fáciles de implementar internamente desde la Facultad, pueden quedar recogidas en un documento de orientaciones dirigidas al tutor, disponibles en el curso virtual del máster. Por ejemplo, la elaboración de unas rúbricas de evaluación especialmente diseñadas para la defensa oral. Así pues, una vez terminado el proceso de análisis, esperamos que los resultados de este trabajo repercutan tanto en la calidad de los trabajos realizados como en la optimización de la tarea de tutorización de los profesores.

Sin embargo, existen dos tipos de quejas recurrentes entre los docentes que no son tan fácilmente subsanables, al menos ni a nivel interno ni a corto plazo: una enfocada hacia su propia institución y otra hacia los estudiantes. Entre las primeras se encuentra la gestión del tiempo (número máximo de TFM y número de horas que cada profesor dedica a un estudiante) y de los plazos de ejecución del trabajo, que desemboca en una saturación del profesorado y una frustración de los estudiantes. Por ello, la universidad debería reflexionar sobre esta carga de trabajo entre su profesorado y la organización del trabajo en plazos tan cortos (un cuatrimestre) para sus estudiantes. Además, los profesores solicitan la adquisición de herramientas institucionales gratuitas antiplagio, una gestión plenamente digital y la reducción de la carga administrativa. Estas propuestas de mejora escapan a comportamientos individuales de los docentes o de la propia facultad.

Entre las exigencias de los profesores hacia los estudiantes, se espera que un alumno que está realizando un máster llegue a la etapa de investigación sabiendo buscar bibliografía, con una redacción académica y capacidad de síntesis propias del registro formal y con una metodología adecuada para el estudio filológico. En definitiva, los profesores piden más autonomía y más rigor académico a los estudiantes. Esta conclusión es especialmente interesante, porque las propuestas de mejora docente siempre se enfocan hacia el profesor y no se suelen analizar las sus opiniones.

Finalmente, atendiendo a las características específicas de nuestra universidad y su repercusión en la elaboración de los TFM (trabajos a distancia realizados por estudiantes que son adultos y además trabajan en muchas ocasiones), parece que el hecho de que la mayoría de estudiantes no viva en Madrid (la distancia) no es un problema a la hora de acceder a la bibliografía, mientras que nos han llamado la atención algunas de las carencias detectadas en la formación previa de los estudiantes (autonomía, redacción formal, etc.), ya que al ser un estudiante adulto, integrado en el mercado laboral o con otros estudios superiores, este tipo de carencias no deberían presentarse.

\section{Referencias bibliográficas}

Cabrera, Nati, Ana Rodera y Albert Sangrà (2012): "Desarrollar y evaluar competencias a través del Trabajo Final de Master. Marco conceptual y guías didácticas", Revista del Congrés Internacional de Docència Universitària i Innovació (CIDUI), 1(1). http://cidui. org/revistacidui/index.php/cidui/article/viewFile/400/394 [consulta: 11 de julio de 2018] 
Da Cunha, Iria (2016): El trabajo de fin de grado y de máster: Redacción, defensa y publicación, Barcelona, UOC.

Fernández Sánchez, José Antonio, et al. (2015): Análisis del proceso de elaboración y tutorización de los TFG y TFM, Alicante, Universidad de Alicante [en línea]. http://rua.ua.es/ dspace/handle/10045/49874 [consulta: 11 de julio de 2018]

González-Calero Somoza, José Antonio, y Pilar Turégano Moratalla (2011): Reflexiones sobre el desarrollo y la evaluación de Trabajos Fin de Máster en el Máster Universitario en Profesor de Educación Secundaria Obligatoria y Bachillerato en la especialidad de matemáticas por la UCLM. Libro de actas [en línea]. https://www.uclm.edu/organos/ vic_docencia/uie/intercampus/intercampusVII/pdf/LibroActasVII_Intercampus.pdf\#page $=73$. [consulta: 11 de julio de 2018]

Miraflores Gómez, Emilio, et al. (2016). La rúbrica como herramienta de evaluación del Trabajo Fin de Máster de Formación del Profesorado de Educación Secundaria y Bachillerato, Formación profesional y Enseñanza de idiomas (especialidad de Educación Física), Madrid, Universidad Complutense de Madrid [en línea]. http://eprints.sim.ucm. es/35930/ [consulta: 11 de julio de 2018]

Pérez Navarro, Antoni, et al. (2013): Guía para diseñar un Trabajo Final de Máster, febrero 2013, Barcelona, UOC. http://openaccess.uoc.edu/webapps/o2/handle/10609/21781 [consulta: 11 de julio de 2018]

Rodríguez, M. Luisa, y Juan Llanes (coords.) (2013). Cómo elaborar, tutorizar y evaluar un Trabajo Fin de Máster, Barcelona, Agencia per a la Qualitat del Sistema Universitari de Catalunya. www.aqu.cat/doc/doc_18533565_1.pdf [consulta: 11 de julio de 2018]

San Mateo Valdehíta, Alicia, María Ángeles Escobar Álvarez y Rubén Chacón Beltrán (en prensa): "El Trabajo Fin de Máster (TFM) de Humanidades en el EEES: Análisis de las necesidades de los estudiantes", REDU. Revista de Docencia Universitaria.

Velayos Martínez, Isabel, et al. (2016): Metodología para tutorización y elaboración de trabajos de fin de grado en las líneas de Derecho procesal propuestas en las titulaciones-EEES de la UA, Alicante, Universidad de Alicante [en línea]. http://rua.ua.es/dspace/ handle/10045/57136 [consulta: 11 de julio de 2018] 